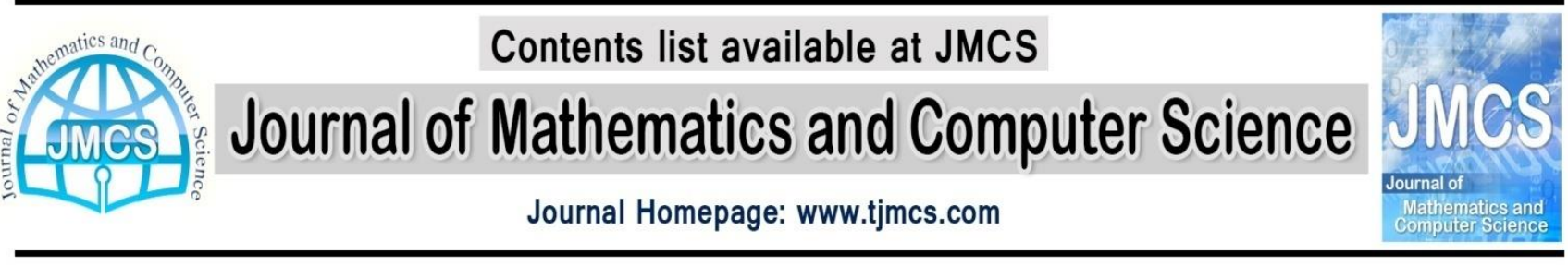

\section{The Biennial Malmquist Index in the of Negative Data}

\author{
Narjes Mohammadi ${ }^{1}$, Alireza Yousefpour ${ }^{*}, 2$ \\ 1. Science and Research branch, Islamic Azad university, Mazandaran, Iran. \\ 2. Department of IT \& Computer, Islamic Azad University of Qaemshahr branch, Iran \\ aryousefpour@yahoo.com
}

Article history:

Received May 2014

Accepted June 2014

Available online July 2014

\begin{abstract}
We purpose the range directional model (RDM), a particular case of the directional distance function, is used for computing efficiency in the presence of negative data. We use RDM efficiency measures to arrive at a Malmquist-type index which can reflect productivity change.

We illustrate how the biennial Malmquist index can be used, not only for comparing the performance of a unit in two time periods, but also for comparing the performance of two different units at the same or different time periods. The proposed approach is then applied to a sample of bank branches where negative data were involved. In this paper, we introduce a biennial Malmquist index of productivity change that can be used with negative data.
\end{abstract}

\section{Introduction}

The computation of productivity change by means of efficiency measures was introduced by Caves et al. (1982) at the first time and developed by Nishimizu and Page (1982) and by Fare et al. (1994), in the context of parametric and non-parametric efficiency measurement, respectively. The Fare et al. (1994) approach has become known as the measurement of productivity change through Malmquist indices. Though several applications of Malmquist indices exist in the literature, to the authors' knowledge there is none where efficiency measures were computed for situations where some data were negative. Negative data may arise due to the use of input-output variables like changes in clients or accounts from one period to the next in the case of our bank branches, or due to the use of variables like profit that may take both positive and negative values. 
The use of profit measures is very common in the banking literature in particular for measuring profit efficiency. To measure efficiency under negative data we use the approach developed by Portela et al. (2004) named range directional model (RDM). To calculate Malmquist indices using the RDM we adapt the Global Malmquist index of Pastor and Lovell (2005), analyzed and extended in Portela and Thanassoulis (2008). The index uses a single reference frontier drawn on a pooled panel of data. We refer to our productivity index as the biennial Malmquist index since the frontier of a pooled panel is often referred to as a biennial frontier. We use the biennial Malmquist index not only to assess the change in the productivity of a unit over time but to also compare the productivities of two units operating at the same or different points in time.

The biennial Malmquist productivity index that has three attractive features: it avoids linear programming infeasibilities under variable returns to scale, it allows for technical regress, and it does not need to be recomputed when a new time period is added to the data set.

\section{DEA Malmquist productivity index}

Fare et al. (1992) construct the DEA-based Malmquist productivity index as the geometric mean of two Malmquist productivity indexes of Caves et al. (1982), which are defined by a distance Function $\mathrm{D}$ (.). Caves et al. (1982) assume $D^{K}\left(x^{k}, y^{k}\right)=1$, that is, they assume the technology for firm $\mathrm{k}$ is efficient, and their distance function does not reveal inefficiency. By allowing for inefficiency and modeling the technology frontier as piecewise linear, Fare et al. (1992) decompose their Malmquist productivity index into two components, one measuring the change in efficiency and the other measuring the change in the frontier technology. The frontier technology determined by the efficient frontier is estimated using DEA for a set of DMUs. However, the frontier technology for a particular DMU under evaluation is only represented by a section of the DEA frontier or a facet. Suppose we have a production function in time period $t$ as well as period $t+1$. Malmquist index calculation requires two single period and two mixed period measures. The two single period measures can be obtained by using the CCR DEA model.

$$
\begin{array}{ll} 
& D_{\circ}^{t}\left(x_{\circ}^{t}, y_{\circ}^{t}\right)=\min \theta \\
\text { s.t } \quad & \sum_{j=1}^{n} \lambda_{i} x_{i j}^{t} \leq x_{i o}^{t}, i=1, \ldots, n \\
& \sum_{j=1}^{n} \lambda_{i} y_{r j}^{t} \geq y_{r o}^{t} / \theta, r=1, \ldots, s \\
& \lambda_{j} \geq 0, \quad j=1, \ldots, n
\end{array}
$$

Where $x_{i o}^{t}$ is the $i^{t h}$ input and $y_{r o}^{t}$ is the $r^{\text {th }}$ output for DMU in time period $t$. The efficiency $\left(D_{o}{ }^{t}\left(x_{o}{ }^{t}, y_{o}{ }^{t}\right)=\theta_{o}^{*}\right)$ determines the amount by which observed inputs can be proportionally reduced, while still producing the given output level. Using $t+1$ instead of $t$ for the above model, we get $\left(D_{o}{ }^{t+1}\left(x_{o}{ }^{t+1}, y_{o}{ }^{t+1}\right)\right.$, the technical efficiency score for DMU in time period $t+1$.

The first of the mixed period measures, which is defined as $\left(D_{o}{ }^{t}\left(x_{o}{ }^{t+1}, y_{o}{ }^{t+1}\right)\right.$ for each $\mathrm{DMU}_{o}$, $o \in Q\{1,2, \ldots, n\}$, is computed as the optimal value to the following linear programming problem: 


$$
\begin{array}{ll} 
& \min \theta \\
\text { s.t } & \sum_{j=1}^{n} \lambda_{i} x_{i j}^{t} \leq x_{i o}^{t+1} \quad, i=1, \ldots, n \\
& \sum_{j=1}^{n} \lambda_{i} y_{r j}^{t} \geq y_{r o}^{t+1} / \theta, r=1, \ldots, s \\
& \lambda_{j} \geq 0, \quad j=1, \ldots, n
\end{array}
$$

Similarly, the other mixed period measure, $D_{o}^{t+1}\left(x_{o}^{t}, y_{o}^{t}\right)$, which is needed in the computation of the output-oriented Malmquist productivity index, is the optimal value to the following linear problem:

$$
\begin{array}{ll} 
& \min \theta \\
\text { s.t } \quad & \sum_{j=1}^{n} \lambda_{i} x_{i j}^{t+1} \leq x_{i o}^{t+1}, i=1, \ldots, n \\
& \sum_{j=1}^{n} \lambda_{i} y_{r j}^{t+1} \geq y_{r o}^{t+1} / \theta, r=1, \ldots, s \\
& \lambda_{j} \geq 0, \quad j=1, \ldots, n
\end{array}
$$

Fare et al, (1992) input-oriented Malmquist productivity index, which measures the productive change of a particular $\mathrm{DMU}_{o}, o \in Q\{1,2, \ldots, n\}$, in time $t+1$ and $t$ is given as

$$
M_{\circ}=\sqrt{\frac{D_{\circ}^{t}\left(x_{\circ}^{t+1}, y_{\circ}^{t+1}\right)}{D_{\circ}^{t}\left(x_{\circ}^{t}, y_{\circ}^{t}\right)} \cdot \frac{D_{\circ}^{t+1}\left(x_{\circ}^{t+1}, y_{\circ}^{t+1}\right)}{D_{\circ}^{t+1}\left(x_{\circ}^{t}, y_{\circ}^{t}\right)}}
$$

It can be seen that the above measure actually is the geometric mean of two Caves et al. (1982) Malmquist productivity indexes. Thus, following Caves et al. (1982) suit, Fare et al. (1992) defined that $M_{O}>1$ indicates productivity gain; $M_{O}<1$ indicates productivity loss; and $\mathrm{M}_{O}=1$ means no change in productivity from time $t$ to $t+1$.

Relaxing Caves et al. (1982) assumption that $\left(D_{o}{ }^{t}\left(x_{o}{ }^{t}, y_{o}{ }^{t}\right)\right.$ and $\left(D_{o}{ }^{t+1}\left(x_{o}{ }^{t+1}, y_{o}{ }^{t+1}\right)\right.$ should equal to one, and allowing for technical inefficiency, Fare et al. (1992) decompose their Malmquist productivity index into two components:

$$
M_{\circ}=\sqrt{\frac{D_{\circ}^{t}\left(x_{\circ}^{t+1}, y_{\circ}^{t+1}\right)}{D_{\circ}^{t}\left(x_{\circ}^{t}, y_{\circ}^{t}\right)} \cdot \frac{D_{\circ}^{t+1}\left(x_{\circ}^{t+1}, y_{\circ}^{t+1}\right)}{D_{\circ}^{t+1}\left(x_{\circ}^{t}, y_{\circ}^{t}\right)}}=\frac{D_{\circ}^{t+1}\left(x_{\circ}^{t+1}, y_{\circ}^{t+1}\right)}{D_{\circ}^{t}\left(x_{\circ}^{t}, y_{\circ}^{t}\right)} \cdot \sqrt{\frac{D_{\circ}^{t}\left(x_{\circ}^{t+1}, y_{\circ}^{t+1}\right)}{D_{\circ}^{t+1}\left(x_{\circ}^{t+1}, y_{\circ}^{t+1}\right)} \cdot \frac{D_{\circ}^{t}\left(x_{\circ}^{t}, y_{\circ}^{t}\right)}{D_{\circ}^{t+1}\left(x_{\circ}^{t}, y_{\circ}^{t}\right)}}
$$

The first component $T E C_{\circ}=\frac{D_{\circ}^{t+1}\left(x_{\circ}^{t+1}, y_{\circ}^{t+1}\right)}{D_{\circ}^{t}\left(x_{\circ}^{t}, y_{\circ}^{t}\right)}$ measures the change in technical efficiency. The second component $F S_{\circ}=\sqrt{\frac{D_{\circ}^{t}\left(x_{\circ}^{t+1}, y_{\circ}^{t+1}\right)}{D_{\circ}^{t+1}\left(x_{\circ}^{t+1}, y_{\circ}^{t+1}\right)} \cdot \frac{D_{\circ}^{t}\left(x_{\circ}^{t}, y_{\circ}^{t}\right)}{D_{\circ}^{t+1}\left(x_{\circ}^{t}, y_{\circ}^{t}\right)}}$ measures the technology frontier shift between time period $t$ and $\mathrm{t}+1$. Fare et al. $(1992,1994 \mathrm{a})$ point out that a value of $F S_{O}$ greater than one indicates a positive 
shift or technical progress, a value of $F S_{O}$ less than one indicates a negative shift or technical regress, and value of $F S_{O}$ equal to one indicates no shift in technology frontier.

\section{The RDM for computing efficiency measures when some data are negative}

The Range Directional Model (RDM) developed in Portela et al. (2004) was inspired by the well known directional distance model of Chambers et al. (1996, 1998). The RDM provides efficiency scores similar in meaning to radial efficiency scores, which can be directly used to compare production units when some inputs and/or outputs are negative. Consider for DMU $j(\mathrm{j}=1, \ldots, \mathrm{n})$ a vector $x_{j}^{t}=\left(x_{1 j}^{t}, \ldots, x_{m j}^{t}\right)$ reflecting $m$ inputs consumed for producing a vector of $s$ outputs $y_{j}^{t}=\left(y_{1 j}^{t}, \ldots, y_{s j}^{t}\right)$ in time period $t \quad(t=1, \ldots, \mathrm{T})$. Define the technology of time period $t$ as $T^{t}=\left\{\left(x^{t}, y^{t}\right)\right.$, $x^{t}$ can produce $\left.y^{t}\right\}$ Considering $\left(g_{x}, g_{y}\right)=\left(g_{x_{1}^{t}}, g_{x_{r}^{t}}, \ldots, g_{x_{m}^{t}}, g_{y_{1}^{t}}, \ldots, g_{y_{s}^{t}}\right)$ as the directional vector, results in the directional distance function being generally defined for a DMU $\mathrm{k}$ as:

$$
\left.\vec{D}^{t}\left(x_{k}^{t}, y_{k}^{t}, g_{x}, g_{y}\right)=\sup \left\{x_{k}^{t}-\beta g_{x}, y_{k}^{t}+\beta g_{y}\right) \in T^{t}\right\}
$$

The directional distance function can be used with any directional vector. Often the observed input and output levels of the DMU concerned are used as the directional vector so as to link with Farrell measures of efficiency. However, using such directional vectors poses a problem when some of its components are negative. This is because the negative components in the directional vector lead to worse rather than better values for the input or output concerned when a positive step length is taken in the direction of the vector. One way to overcome this problem would be to use a fixed directional vector, like the unit direction vector used in or the average input and output vector used in Park and Weber (2006). However, the choice of a fixed direction would be arbitrary, while our approach selects a direction that has an intuitive appeal, because we can control whether improvements are sought which lead to attainment targets that are either least or alternatively most challenging to attain.

In the RDM the directional vector reflects ranges of possible improvement defined for DMU $\mathrm{k}$, and for a given time period $\mathrm{t}$, as

$$
g_{y_{r k}^{t}}=R_{y_{r k}^{t}}=\max _{j}\left\{y_{r j}^{t}\right\}-y_{r k}^{t}, \quad r=1 \ldots s \text { and } g_{x_{i k}^{t}}=R_{x_{i k}^{t}}=x_{i k}^{t}-\min \left\{x_{i j}^{t}\right\}, i=1, \ldots, m
$$

These ranges assume implicitly the existence of an ideal point with maximum outputs and minimum inputs observed in period t. Note that this idea has also been used in a different context by Fare et al. (2004), where a hypothetical DMU with maximum outputs and minimum inputs was used as a base against which all others were compared in a cross section of countries in one year. The RDM for $\mathrm{DMU}_{\mathrm{k}}$, observed in time period $\mathrm{t}$, is as in

$$
\left.\vec{D} R^{t}\left(x_{k}^{t}, y_{k}^{t}, R_{x_{k}^{t}}, R_{y_{k}^{t}}\right)=\sup \left\{\beta \mid x_{k}^{t}-\beta R_{x_{k}^{t}}, y_{k}^{t}+\beta R_{y_{k}^{t}}\right) \in T^{t}\right\}
$$

In this paper we are particularly interested in the output oriented RDM model (that is used in the empirical application). This is a particular case of the general model in (2), where $R_{x_{k}^{t}}$ is set to the zero vector. For the case of a technology exhibiting variable returns to scale (VRS) the output oriented RDM can be solved through linear programming using the model in (3), for each $D_{M} U_{k}$ where (k $=1, \ldots, \mathrm{n}$ ) observed in a general time period $t$.

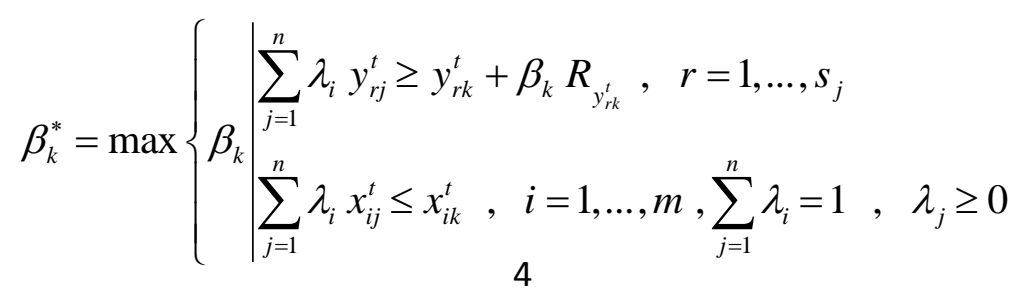


The optimum solution to model (3) provides an inefficiency measure equal to $\beta_{k}^{*}=\vec{D} R^{t}\left(x_{k}^{t}, y_{k}^{t}, 0, R_{y_{r k}^{t}}\right)$. This measure represents proportion of the range vector $R_{y_{r k}^{t}}$ by which the outputs of unit $k$ should be increased so that it will reach the frontier. When $\beta_{k}^{*}=0$ the unit is on the frontier. Otherwise $R D M^{t}\left(x_{k}^{t}, y_{k}^{t}, \cdot, R_{y_{k}^{t}}\right)=1-\vec{D} R^{t}\left(x_{k}^{t}, y_{k}^{t}, \cdot, R_{y_{k}^{t}}\right)=1-\beta_{k}^{*}$ is a measure of the output efficiency of unit $k$. Note that we have $1-\beta_{k}^{*}=\frac{\max _{j}\left\{y_{r j}^{t}\right\}-y_{r k}^{t *}}{\max _{j}\left\{y_{r j}^{t}\right\}-y_{r k}^{t}}$ for each output $r$ for which the output constraint in (3) is binding, $y_{r k}^{t *}$ being the value at the frontier (target) yielded by (3) for output $\mathrm{r}$ (i.e $\left.y_{r k}^{t *}=\sum_{j} \lambda_{j}^{*} y_{r j}^{t}\right)$.the measure is radial as in the traditional DEA model in the sense that it leads to target output levels $y_{r k}^{t *}$ for $D M U_{k}$ which are an equiproportional distance $\left(1-\beta_{k}^{*}\right)$ from the observed output levels $\left(y_{r k}^{t *}\right)$. However, the proportions here are not of the distance between the observed output levels and the origin as in the traditional DEA model, but rather of the distance between the observed and the ideal output levels. In other words the ideal point here plays the role of the origin in traditional radial DEA measures of efficiency.

Note, however, that there is qualitative difference between measuring efficiency relative to observed benchmark units, as in traditional DEA, and the use of an ideal point to simply define a direction of improvement. For example, the direction of improvement may not necessarily take us towards benchmark units that have a similar mix of inputs and outputs to the unit being assessed. It is shown in Portela et al. (2004) that the RDM model is translation and units invariant when defined for a variable returns to scale (VRS) technology. A constant returns to scale (CRS) assumption for the technology is not consistent with the existence of negative data, because a CRS technology assumes that any activity can be "radially expanded or contracted to form other feasible activities". However, the radial scaling loses its meaning when we have a mix of positive and negative data, since a radial expansion of the absolute value of a negative factor would make its level worse (i.e. less desirable). Hence the CRS assumption that efficiency is maintained under the same radial expansion or contraction of all inputs and outputs cannot be guaranteed. In addition, when some data are negative one may not be able to define an efficient frontier passing through the origin, as is assumed under CRS, and therefore an assumption of global CRS breaks down for negative data. The ability of the RDM to yield radial-like measures of efficiency in the presence of negative data makes this model suitable for computing Malmquist indices when some data are negative.

\section{Biennial Malmquist indices}

As noted earlier, for computing our index and indicator of productivity change we shall use a biennial frontier, which envelops the pooled data of a panel covering a number of time periods, to which we refer collectively as the meta-period. In the present context, one advantage of using biennial frontiers that we can handle well VRS technologies which become necessary in the presence of negative data. Using biennial meta-frontiers under VRS makes it possible to compute the index for all units. It is recalled that some approaches to decomposing Malmquist indices of productivity change under VRS can encounter infeasible models for some units.

In the following we consider output-oriented distance functions and Malmquist indices and a balanced panel of $\mathrm{j}=1, ., \mathrm{n}$ producers in each of $\mathrm{t}=1, ., \mathrm{u}$ time periods. Denote by $(x, y) \in R_{+}^{m} \times R_{+}^{s}$ the inputoutput vector of a generic producer and by $\left(x^{t}, y^{t}\right) \in R_{+}^{m} \times R_{+}^{s}$ the corresponding vector for a specific 
producer $j$ in time period $t$. For each t consider two benchmark technologies, the period $t$ technology defined as

$$
T_{c}^{t}=\left\{(X, Y) \in R_{+}^{m+s} \mid X \geq \sum_{j=1}^{n} \lambda^{t}{ }_{j} X^{t}{ }_{j}, Y \leq \sum_{j=1}^{n} \lambda^{t}{ }_{j} Y^{t}{ }_{j}, \lambda^{t}{ }_{j} \geq 0, j=1, \ldots, n\right\}
$$

And the technology associated with the subsequent period, $T_{c}^{t+1}$ defined similarly. Based on these two technologies the base $\mathrm{t}$ biennial technology $T_{c}^{B}$ can be defined as the convex hull of the period $t$ and period $t+1$ technologies $T_{c}^{B}=\operatorname{con}_{\mathrm{v}}\left\{T_{c}^{t}, T_{c}^{t+1}\right\}$. The subscript $c$ in $T_{c}^{K}, K=t, t+1$ indicates that $T_{c}^{K}$ exhibits constant returns to scale (CRS), $\lambda T_{c}^{K}=T_{c}^{K}$ for all $\lambda>0$. Hence $T_{c}^{B}$ also satisfies CRS.

The biennial technology corresponds to a DEA window analysis with a window width of two. In the case of panel data consisting of two time periods, i.e. $u=2$, the biennial technology is identical to a pooled or meta-frontier technology also used to construct a global Malmquist index. But in the general case of more than two time periods $(\mathrm{u}>2)$, a series of $u$ - 1 overlapping biennial technologies exists for each pairwise comparison of adjacent time periods. The biennial Malmquist index is defined specifically for the adjacent time periods $t$ and $t+1$ since two adjacent time periods are sufficient to establish the desirable properties of avoiding infeasibility, allowing technical regress, and maintaining previous productivity calculations. Sufficiency of two time periods for these desirable properties does not preclude the construction of a triennial Malmquist index. However the biennial Malmquist index is not transitive because it is constructed from a series of overlapping two period technologies, and these technologies can differ. This drawback, however, is not uncommon; it is shared by all Malmquist indices except the global index, which is transitive because it contains a single technology. Based on the classic CRS output distance function for (x, y) defined on the period $t$ technology, $D_{c}^{t}(x, y)=\min \left\{Q>0 \mid(x, y / Q) \in T_{c}^{t}\right\}$ the standard output-oriented adjacent period $t$ Malmquist index for producer $\mathrm{j}$ is given by

$$
M_{c}^{t}\left(x_{j}^{t}, y_{j}^{t}, x_{j}^{t+1}, y_{j}^{t+1}\right)=\frac{D_{c}^{t}\left(x_{j}^{t+1}, y_{j}^{t+1}\right)}{D_{c}^{t}\left(x_{j}^{t}, y_{j}^{t}\right)}
$$

And the adjacent period $t+1$ Malmquist index is defined similarly, using the output distance function defined on the technology for period $t+1, D_{c}^{t+1}, M_{c}^{t+1}$ likely differs from $M_{c}^{t}$, which leads to the definition of the adjacent Malmquist productivity index, $M_{c}$, as the geometric mean of $M_{c}^{t}$ and $M_{c}^{t+1}$

$$
M_{c}=\left(\frac{M_{c}^{t}\left(x_{j}^{t}, y_{j}^{t}, x_{j}^{t+1}, y_{j}^{t+1}\right)}{M_{c}^{t+1}\left(x_{j}^{t}, y_{j}^{t}, x_{j}^{t+1}, y_{j}^{t+1}\right)}\right)^{\frac{1}{2}}
$$

i.e. similarly to the definition of $D_{c}^{t}$, wedefine the biennial output distance function $D_{c}^{B}$, based on $T_{c}^{B}$ instead of $T_{c}^{t}$, i.e. $D_{c}^{t}(x, y)=\min \left\{Q>0 \mid\left(x, y / Q^{\prime}\right) \in T_{c}^{t}\right\}$. We further define the biennial CRS Malmquist index for producer $j$ as

$$
M_{c}^{B}\left(x_{j}^{t}, y_{j}^{t}, x_{j}^{t+1}, y_{j}^{t+1}\right)=\frac{D_{c}^{B}\left(x_{j}^{t+1}, y_{j}^{t+1}\right)}{D_{c}^{B}\left(x_{j}^{t}, y_{j}^{t}\right)}
$$


Since we are using the biennial CRS technology, which includes both the period $t$ and period $t+1$ technologies, we do not need to resort to any geometric mean when defining (6).

The CRS benchmark technologies should be distinguished from the best practice technologies allowing for variable returns to scale (VRS). This convention enables it [the Malmquist index] to incorporate the influence of scale economies as a departure of the best practice technology from the benchmark technology. To define VRS counterparts of the CRS constructs above, consider first the period $t$ VRS technology defined as

$$
T_{v}^{t}=\left\{(X, Y) \mid X \geq \sum_{j=1}^{n} \lambda_{j}^{t} X^{t}{ }_{j}, Y \leq \sum_{j=1}^{n} \lambda_{j}^{t} Y^{t}{ }_{j}, \lambda^{t}{ }_{j}=1, j=1, \ldots, n\right\}
$$

There is only difference between $T_{c}^{t}$ and $T_{c}^{v}$ that the latter includes the convexity constraint on the lambdas. Similarly the remaining VRS technologies are easily defined and denoted by the subscript " $v$ " rather than " $c$ ". Hence the adjacent VRS Malmquist index is given by

$$
M_{v}=\left(\frac{M_{v}^{t}\left(x_{j}^{t}, y_{j}^{t}, x_{j}^{t+1}, y_{j}^{t+1}\right)}{M_{v}^{t+1}\left(x_{j}^{t}, y_{j}^{t}, x_{j}^{t+1}, y_{j}^{t+1}\right)}\right)^{\frac{1}{2}}
$$

And the biennial VRS Malmquist index is defined by

$$
M_{v}^{B}\left(x_{j}^{t}, y_{j}^{t}, x_{j}^{t+1}, y_{j}^{t+1}\right)=\frac{D_{v}^{B}\left(x_{j}^{t+1}, y_{j}^{t+1}\right)}{D_{v}^{B}\left(x_{j}^{t}, y_{j}^{t}\right)}
$$

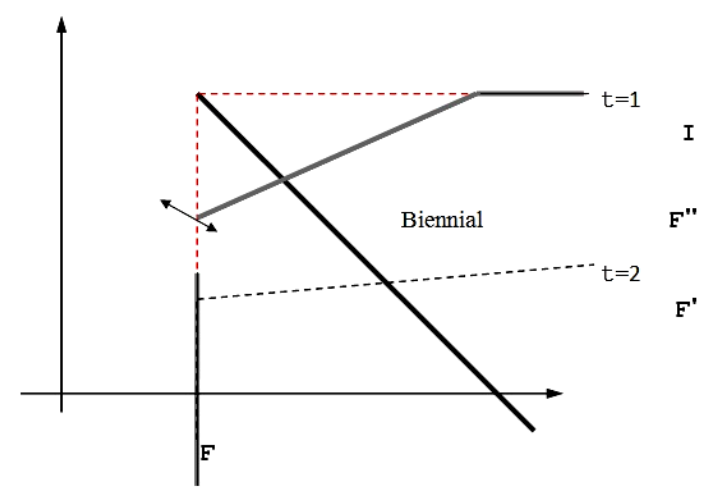

Fig.1 Illustration of the biennial Malmquist index

In Fig. 1 consider two period specific frontiers $(t$ and $t+1)$ and a biennial-frontier (lying above the period $t$ and $t+l$ frontiers for ease of illustration).

Branch $F$ observed in period $t$ has a RDM efficiency of IF/IF when it is assessed in relation to the period $t$ frontier. We can also assess the efficiency of branch $F$ in relation to the biennial meta-frontier, which we refer to as biennial efficiency. The biennial efficiency of branch $F$ is given by IF"/IF, and it can be decomposed into two components: The within-period-efficiency (IF'/IF) and a technological gap(IF"/IF'). That is, $I F^{\prime \prime} / I F=I F^{\prime} / I F \times I F^{\prime \prime} / I F^{\prime}$. The within-period-efficiency measures how distant the production unit is from the frontier of the period in which it was observed. The technological gap (TG) measures the distance between the period $t$ frontier and the biennial frontier, at the input/output mix of the unit concerned. 
Generalising, let $\vec{D} R^{t}\left(x_{j}^{t}, y_{j}^{t}, 0, R_{y_{j}^{t}}\right)$ (defined in (2)) be expressed as $\vec{D} R^{B f}\left(x_{j}^{t}, y_{j}^{t}, 0, R_{y_{j}^{t}}^{B f}\right)$ when the technology used for computing the directional distance function of DMU $j$ in period $t$ is the biennial frontier. The superscript Bf on DR indicates the distance function is in relation to the metafrontier, while the superscript $\mathrm{Bf}$ on $\mathrm{R}$ indicates that the ideal point for computing the range $\mathrm{R}$ is a global ideal point defined over the biennial period. Thus the ideal point $I_{r}=\max _{t}\left\{\max _{j}\left\{y_{r j}^{t}\right\}\right.$, for each output $(\mathrm{r}=1, . ., \mathrm{s})$. We define $R D M^{B f}\left(x_{j}^{t}, y_{j}^{t}, 0, R_{y_{j}^{t}}^{B f}\right)=1-\vec{D} R^{B f}\left(x_{j}^{t}, y_{j}^{t}, 0, R_{y_{j}^{t}}^{B f}\right)$ as the RDM output 'biennial efficiency' of unit $j$ in period $t$. Further, let $R D M^{t}\left(x_{j}^{t}, y_{j}^{t}, 0, R_{y_{j}^{t}}^{B f}\right)=1-\vec{D} R^{t}\left(x_{j}^{t}, y_{j}^{t}, 0, R_{y_{j}^{t}}^{B f}\right)$ be the RDM 'within- period-efficiency' of unit $\mathrm{j}$ observed in period $\mathrm{t}$ and computed using model (3) with technology of period $\mathrm{t}$ and with reference to the global ideal point defined above (Note that the within-period-efficiency is computed with reference to a global ideal point rather than to a within period ideal point, and in that respect it cannot be considered as a 'pure' within period measure.

Retaining the same ideal point for within-period efficiencies and for biennial efficiencies makes the vector that departs from the observed point to the global ideal point collinear with the vector that departs from the target (on the within-period frontier) to the ideal point. This collinearity allows the meaningful computation of ratios between the various RDM efficiency measures as is now explained. Thus, we have:

$$
R D M^{B f}\left(x_{j}^{t}, y_{j}^{t}, 0, R_{y_{j}^{t}}^{B f}\right)=R D M^{t}\left(x_{j}^{t}, y_{j}^{t}, 0, R_{y_{j}^{t}}^{B f}\right) \times T G_{j}^{t}
$$

Is retrieved residually as

$$
T G_{J}^{t}=R D M^{B f}\left(x_{j}^{t}, y_{j}^{t}, 0, R_{y_{j}^{t}}^{B f}\right) / R D M^{t}\left(x_{j}^{t}, y_{j}^{t}, 0, R_{y_{j}^{t}}^{B f}\right)
$$

Using the above definitions where efficiency measures are computed through the RDM model, we can define a biennial Malmquist index as:

$$
B M_{j}^{t, t+1}=\frac{R D \vec{M}^{B f}\left(x_{j}^{t+1}, y_{j}^{t+1}, \circ, R_{y_{j}{ }^{t+1}}^{B f}\right)}{R D \vec{M}^{B f}\left(x_{j}^{t}, y_{j}^{t}, \circ, R_{y_{j}{ }^{t}}^{B f}\right)}
$$

When $B M_{j}^{t, t+1}$ is greater than 1, the productivity of unit $j$ has improved from $t$ to $t+1$ (since its biennial efficiency in period $t+1$ is higher than that in $t$ ). Productivity has declined when $B M_{j}^{t, t+1}$ is below 1. In $B M_{j}^{t, t+1}$ we used two subsequent periods ( $t$ and $\left.t+1\right)$, but the definitions in (11) and throughout the paper are valid whatever the two periods being compared. Using (9) we can decompose the biennial Malmquist index as shown in (12).

$$
B M_{j}^{t, t+1}=\frac{R D \vec{M}^{B f}\left(x_{j}^{t+1}, y_{j}^{t+1}, \circ, R_{y_{j}^{t+1}}^{B f}\right)}{R D \vec{M}^{B f}\left(x_{j}^{t}, y_{j}^{t}, \circ, R_{y_{j}^{t}}^{B f}\right)}=\frac{R D \vec{M}^{t+1}\left(x_{j}^{t+1}, y_{j}^{t+1}, \circ, R_{y_{j}^{t+1}}^{B f}\right)}{R D \vec{M}^{t}\left(x_{j}^{t}, y_{j}^{t}, \circ, R_{y_{j}^{t}}^{B f}\right)} \times \frac{T G_{j}^{t+1}}{T G_{j}^{t}}
$$

The first term in (12) captures the pure technical efficiency change of unit $j$ from year $t$ to year $t+1$. 


\section{Empirical application to bank branches}

We consider input and output five bank branches in period $t$ and period $t+1$ and to use biennial Malmquist in the model RDM to arrive at efficiency measure input and output in period $t$ and period $t+1$.

\begin{tabular}{ccccccc}
\hline Bank & Input 1 & Input 2 & Input 3 & Output 1 & Output 2 & Output 3 \\
\hline A & 1639.17 & 14.21 & 16675 & $\mathbf{8 6 6 3}$ & $\mathbf{2 0 3 5 3 8}$ & $\mathbf{3 5 4 . 9 8}$ \\
\hline B & 1307.91 & 12.15 & 7438 & $\mathbf{1 2 4 4 8 . 2 3}$ & $\mathbf{1 5 7 7 9 6 . 7}$ & $\mathbf{7 2 8 . 1 2}$ \\
\hline C & 981.03 & 13.11 & 10386.79 & $\mathbf{1 2 3 8 . 3}$ & $\mathbf{2 0 2 1 6 1 . 1}$ & $\mathbf{9 3 2 . 3 9}$ \\
\hline D & 659.82 & 15.99 & 9270.5 & $\mathbf{4 3 6 3}$ & $\mathbf{6 5 1 2 3 7}$ & $\mathbf{1 8 3 2 . 7 4}$ \\
\hline E & 2372.7 & 11.13 & 19420 & $\mathbf{4 2 4 0 . 3 3}$ & $\mathbf{5 7 0 8 3 8 . 3 3}$ & $\mathbf{5 8 1 . 2 3}$ \\
\hline
\end{tabular}

Table 1 input and output five bank branches in period $t$

\begin{tabular}{ccccccc}
\hline Bank & Input 1 & Input 2 & Input 3 & Output 1 & Output 2 & Output 3 \\
\hline A & 1532.92 & 13.4 & 16704 & $\mathbf{8 7 0 2}$ & $\mathbf{2 1 5 8 7 3}$ & $\mathbf{3 2 4 3 . 2}$ \\
\hline B & 1319.56 & 12.14 & 7594.35 & $\mathbf{2 3 8 2 . 8 8}$ & $\mathbf{1 5 9 9 0 1 . 0 5}$ & $\mathbf{8 4 1 . 7 9}$ \\
\hline C & 964.92 & 13.01 & 10577.9 & $\mathbf{1 3 5 8 . 6}$ & $\mathbf{2 0 8 1 5 1 . 9}$ & $\mathbf{2 9 0 2 . 5 1}$ \\
\hline D & 630.6 & 15.85 & 8800.5 & $\mathbf{3 8 8 4}$ & $\mathbf{6 0 4 7 8 3 . 5}$ & $\mathbf{2 4 0 3 . 6 2}$ \\
\hline E & 2504.67 & 11.08 & 19401.83 & $\mathbf{4 0 4 3 . 5}$ & $\mathbf{5 9 0 1 2 8 . 5}$ & $\mathbf{1 2 8 9 5 . 6 7}$ \\
\hline
\end{tabular}

Table 2 input and output five bank branches in period $t+1$

To using the data and this model $g_{y_{r k}^{t}}=R_{y_{r k}^{t}}=\max _{j}\left\{y_{r j}^{t}\right\}-y_{r k}^{t}, r=1, . ., s$ Calculations value $R_{y_{r k}^{t}}$ for period $t$ and period $t+1$.

\begin{tabular}{c|cc|} 
& Period $t$ & Period $\boldsymbol{t}+\boldsymbol{1}$ \\
\hline$R_{X 11}$ & 0.0 & 0.0 \\
$R_{X 12}$ & 447.699 & 388910.5 \\
$R_{X 13}$ & 1477.76 & 12571.47 \\
$R_{X 21}$ & 6212.77 & 5419.12 \\
$R_{X 22}$ & 493440.3 & 444882.45 \\
$R_{X 23}$ & 1104.62 & 12053.88 \\
$R_{X 31}$ & 7424.7 & 7343.4 \\
$R_{X 32}$ & 449075.9 & 396631.6 \\
$R_{X 33}$ & 900.35 & 9993.16 \\
$R_{X 41}$ & 4300.0 & 4818.0 \\
$R_{X 42}$ & 0.0 & 0.0 \\
$R_{X 43}$ & 0.0 & 10492.05 \\
$R_{X 51}$ & 4422.67 & 46585.0 \\
$R_{X 52}$ & 80398.67 & 14655.0 \\
$R_{X 53}$ & 1251.51 & 0.0
\end{tabular}

Table 3 value $R_{y_{r k}^{t}}$ for period t and period $\mathrm{t}+1$ 
Table 4 shows optimal values $\beta^{t}$ and $\beta^{t+1}$ is given by model RDM.

\begin{tabular}{|c|cc|}
\multicolumn{1}{c}{} & $\operatorname{Max} \beta^{t}$ & $\operatorname{Max} \beta^{t+1}$ \\
\hline $\mathbf{1}$ & $\mathbf{0 . 5 8 6 3 1 0}$ & $\mathbf{0 . 0}$ \\
$\mathbf{2}$ & $\mathbf{0 . 0}$ & $\mathbf{0 . 1 3 1 2 5 2 8}$ \\
$\mathbf{3}$ & $\mathbf{0 . 6 4 3 2 4 0}$ & $\mathbf{0 . 0}$ \\
$\mathbf{4}$ & $\mathbf{0 . 0}$ & $\mathbf{0 . 0}$ \\
$\mathbf{5}$ & $\mathbf{0 . 2 1 7 4 1 7}$ & $\mathbf{0 . 0}$
\end{tabular}

Table 4 optimal values $\beta^{t}$ and $\beta^{t+1}$

The solution to model RDM provides an efficiency measure is as in:

$$
R D M^{t}\left(x_{k}^{t}, y_{k}^{t}, \cdot, R_{y_{k}^{t}}\right)=1-\vec{D} R^{t}\left(x_{k}^{t}, y_{k}^{t}, \cdot, R_{y_{k}^{t}}\right)=1-\beta_{k}^{*}
$$

A measure of productivity is given by the ratio of efficiency measure in two periods $(t$ and $t+1)$, we can define a biennial Malmquist index as:

$$
\begin{aligned}
& \qquad M_{j}^{t, t+1}=\frac{R D \vec{M}^{B f}\left(x_{j}^{t+1}, y_{j}^{t+1}, \circ, R_{y_{j}^{t+1}}^{B f}\right)}{R D \vec{M}^{B f}\left(x_{j}^{t}, y_{j}^{t}, \circ, R_{y_{j}^{t}}^{B f}\right)}=\frac{1-\beta_{j}^{t+1}}{1-\beta_{j}^{t}} \\
& B M_{1}^{t, t+1}=\frac{1-0}{1-0.586310}=\frac{1}{0.41369}=2.41726896 \\
& B M_{2}^{t, t+1}=\frac{1-0.1312528}{1-0}=\frac{0.8687472}{1}=0.8687472 \\
& B M_{3}^{t, t+1}=\frac{1-0}{1-0.643240}=\frac{1}{0.35676}=2.8030048 \\
& B M_{4}^{t, t+1}=\frac{1-0}{1-0}=\frac{1}{1}=1 \\
& B M_{5}^{t, t+1}=\frac{1-0}{1-0.2174417}=\frac{1}{0.785583}=1.27293996
\end{aligned}
$$

When $B M_{j}^{t, t+1}$ is greater than 1 , the productivity of unit $j$ has improved from to $t+1$ (since its biennial efficiency in period $t+1$ is higher than that in $t$ ). Productivity has declined when $B M_{j}^{t, t+1}$ is below 1 .

$D M U_{1}$ productivity has improved in period $\mathrm{t}$ and period $\mathrm{t}+1 . D M U_{2}$ productivity has declined in period $\mathrm{t}$ and period $\mathrm{t}+1 . \mathrm{DMU}_{3}$ productivity has improved in period $\mathrm{t}$ and period $\mathrm{t}+1$. $D M U_{4}$ productivity has not changed in period $\mathrm{t}$ and period $\mathrm{t}+1 . D M U_{5}$ productivity has improved in period $\mathrm{t}$ and period $\mathrm{t}+1$.

\section{6- Conclusion}

This paper has presented an approach for computing biennial Malmquist indices for measuring productivity change over time and productivity differences between units in multi-input/multi-output contexts where some of those inputs and/or outputs take negative values.

The paper also shows how biennial Malmquist indices can be computed in order to compare units on performance over time. This can be useful in several contexts where a company or government body needs to monitor comparative productivity changes between units. The paper uses unit-specific 
boundaries and the biennial-frontier to compare units on productivity, and decompose the resulting measure into a number of components capturing the position of a unit within its own unit-specific frontier and the differences in unit-specific frontiers relative to the biennial-frontier.

\section{References}

[1] DW. Caves, LR. Christensen, WE. Diewert, The economic theory of index numbers and the measurement of inputs, outputs and productivity (1982).

[2 ] R.G. Chambers, Y. Chung, R. Fare, Benefit and distance functions (1996).

[3 ] R.G. Chambers, Y. Chung, R. Fare, Profit, directional distance functions, and Nerlovian efficiency (1998).

[4] R. Färe, S. Grosskopf, M. Norris, Z. Zhang, Productivity growth, technical progress, and efficiency change in industrialized countries (1994).

[5] R. Fare, S. Grosskopf, F. Hernadez-Sancho, Environmental performance: An index number approach. Resource and Energy Economics 26, 343-352 (2004).

[6 ] M. Nishimizu, J.M. Page, Total factor productivity growth, technological progress and efficiency change (1982).

[7 ] K.H. Park, , W.L. Weber, A note on efficiency and productivity growth in the Korean banking industry, 1992-2002, Journal of Banking and Finance 30, 2371-2386 (2006).

[8 ] J.T. Pastor, C.A.K. Lovell, A global Malmquist productivity index. Economics Letters 88, 266271 (2005).

[9] J.T. Pastor, C.A.K. Lovell, the biennial Malmquist productivity change index (2011).

[10] M.C.A.S. Portela, E. Thanassoulis, G.P.M. Simpson, Negative data in DEA: A directional distance approach applied to bank branches. Journal of the Operational Research Society 55, 1111-1121 (2004).

[11] M.C.A.S. Portela, E. Thanassoulis, Comparative efficiency analysis of Portuguese bank branches. European Journal of Operational Research 177, 1275-1288 (2007).

[12] M.C.A.S. Portela, E. Thanassoulis, A circular Malmquist-type index for measuring productivity. AstonWorking Paper RP08-02., Aston University Birmingham B4 7ET, U (2008). 